REVISTA PROYECCIONES No 11: $1-17$

Julio 1986 - I.S.S.N. 0716-0917

\title{
SOLUCIONES SECCIONALMENTE DIFERENCIABLES EN EL PROBLEMA DUAL DEL PROBLEMA CONTINUO DE TRANSFORTE*
}

EDUARDO CAMINO E. ** OSCAR ROJO J. $* *$

\section{INTRODUCCION.}

En este trabajo presentamos la solución a un problema de Optimización propuesto por R. Bellman [1] o problema dual del problema continuo de transporte [3] cuando el núcleo $k(x, y)=G(y-x)$, donde $G$ es una función cuya segunda derivada satisface $G^{\prime \prime}(0)=0, G^{\prime \prime}(t)>0$ para $t<0 y$ $G^{\prime \prime}(t)<0$ para $t>0$. Los resultados pueden ser extendidos a otros casos análogos. El problema de Bellman en una versión generalizada [3] es: Dado un núcleo continuo $\mathrm{K}(\mathrm{x}, \mathrm{y})$ definido en el rectángulo $[\mathrm{a}, \mathrm{b}] \mathrm{x}[\mathrm{c}, \mathrm{d}$, encon trar un par de funciones continuas $f y g$ definidas en [a, b] y [c, d] respectivamente tal que

$$
f(x)+g(y) \geqslant K(x, y) \text { en }[a, b] x[c, d]
$$

* Resultados parciales de un Proyecto de Investigación, actualmente en ejecución, financiado por la Universidad del Norte. ** Departamento de Matemáticas, Universidad del Norte. 


$$
F(f, g)=\int_{a}^{b} \alpha(x) f(x) d x+\int_{c}^{d} \beta(y) g(y) d y \text { sea mínimo, donde }
$$

las funciones a y $B$ son continuas, positivas y satisfacen

$$
\int_{a}^{b} \alpha(x) d x=\int_{c}^{d} \beta(y) d y
$$

Todo par de funciones que satisfaga la condición (1) es llamado par admisi ble y todo par admisible que minimice la funcional $F$ es llamado par mini mal. Es claro que si $(f, g)$ es un par minimal también lo es el par (f + c, g - c) cualquiera sea la constante c. Entonces, no podemos esperar unicidad en la solución a menos que se introduzca una condición de nor malización:

$$
\int_{a}^{b} f(x) \alpha(x) d x=\int_{C}^{d} g(y) B(y) d y
$$

Una solución completa para el problema de Bellman es dada en [3] cuando $\mathrm{K}$ es un núcleo polinomial y la segunda derivada parcial mixta $\mathrm{K}_{\mathrm{xy}}$ no cambia de signo en el rectángulo [a, b] x [c, d]. Este resultado puede extenderse, usando el mismo argumento, para el caso en que $\mathrm{K}$ es uná función analítica y la misma hipótesis para $\mathrm{K}_{\mathrm{xy}}$ [4]. Este resultado está dado en el siguiente teorema.

\section{Teorema.}

Sea $K$ una función analítica. Supongamos que $k_{x y}$ no cambia de signo en $[\mathrm{a}, \mathrm{b}] \mathrm{x}[\mathrm{c}, \mathrm{d}]$. Sea $\mathrm{y}=\rho(\mathrm{x})$ la ecuación de la diagonal cuya pendiente tiene el signo de $K_{x y}$. Entonces, existe un único par (f,g) mini mal diferenciable para la funcional 


$$
F(f, g)=\int_{a}^{b} f(x) d x+\frac{b-a}{d-c} \int_{c}^{d} g(y) d y
$$

Este par minimal puede ser encontrado integrando

$$
\left.\begin{array}{l}
f^{\prime}(x)=K_{x}(x, \rho(x)) \\
g^{\prime}(y)=K_{y}\left(p^{-1}(y), y\right)
\end{array}\right\}
$$

escogiendo las constantes de integración tal que

$$
f(x)+g(p x))=K(x, p(x))
$$

Observamos que si $[a, b] \times[c, a]$ es un cuarirado entonces la fun cional dada en (5) llega a ser

$$
F(f, g)=\int_{a}^{b} f(x) d x+\int_{C}^{d} g(y) d y
$$

y la condición de normalización se convierte en

$$
\int_{a}^{b} f(x) d x=\int_{c}^{d} g(y) d y
$$

En la siguiente sección veremos que cuando $k(x, y)=G(y-x)$ las conclusiones del teorema antes dado sigien siende inlidás aun cuando $G$ no sea una función analítica.

2. NUCLEOS DE LA FORMA $K(x, y)=G(y-x)$, DONDE $G "$ NO CAMBIA DE SIGNO.

Teorema.

Si $K(x, y)=G(y-x)$, donde $G$ es tal que $G^{\prime \prime}(y-x)$ no cambia de signo en el cuadrado $[a, b] \times[c, d]$, entonces existe un único par minimal di 
ferenciable. Este par puede ser encontradu utilizando las fómulas (6) y (7), donde $y=\rho(x)$ es la ecuación de la diagonal cuya pendiente tiene el signo de $-G^{\prime \prime}$.

Demostración.

Sea $y=\rho(x)=m x+n$ la ecuación de lá diagonal cuya pendiente $m$ tiene el signo de $-G "$. La utilización de las fómmulas dadas en

(6) conduce a

$$
\begin{aligned}
& f^{\prime}(x)=-G^{\prime}((m-1) x+n) \\
& g^{\prime}(y)=G^{\prime}\left(\frac{m-1}{m} y+\frac{n}{m}\right)
\end{aligned}
$$

Distinguimos los casos i) $m=1$ y $\quad$ ii) $m=-1$.

i) Sea $\mathrm{m}=1$. Entonces,

$$
f^{\prime}(x)=-G^{\prime}(n), \quad g^{\prime}(y)=G^{\prime}(n)
$$

Integrando obtenemos $f(x)=-G^{\prime}(n) x+c_{1} y \quad g(y)=G^{\prime}(n) y+c_{2}$. De la ecuación (7) se sigue que las constantes $c_{1} y c_{2}$ deben satisfacer

$$
c_{1}+c_{2}=G(n)-n G^{\prime}(n)
$$

Probamos a continuación que este par de funciones es admisibie, es decir, que

$$
D(x, y)=K(x, y)-f(x)-g(y) \leqslant 0 .
$$

Entonces,

$$
D(x, y)=G(y-x)+G^{\prime}(n) x-G^{\prime}(n) y-C(n)+n G^{\prime}(n)
$$

P.ra $y=x+n$ tenemos $D(x, x+n)=0$. Sea $y \neq x+n$. Aplicando dos ve- 
ces consecutivas el teorema del valor medio obtenemos

$$
\begin{aligned}
D(x, y) & =G^{\prime}(n)(x-y+n)+G(y-x)-G(n) \\
& =G^{\prime}(n)(x-y+n)+G^{\prime}(p)(y-x-n) \\
& =(x-y+n)\left(G^{\prime}(n)-G^{\prime}(p)\right) \\
& =(x-y+n) G^{\prime \prime}(q)(n-p)
\end{aligned}
$$

cierto $p$ entre $(y-x)$ y $n, y$ cierto $q$ entre $n y$. Los factores $(x-y+n)$ y $n-p$ tienen el mismo signo. Entonces, el signo de $D(x, y)$ está dado por el signo de G"(q), el cual es negativo pues en este caso $\mathrm{m}=1$.

ii) Sea $m=-1$. Entonces,

$$
\begin{aligned}
& f(x)=\frac{1}{2} G(n-2 x)+c_{1} \\
& g(y)=\frac{1}{2} G(2 y-n)+c_{2}
\end{aligned}
$$

De la ecuación (7) obtenemos que $c_{1}+c_{2}=0$. Par . = $n-x$ tenemos $D(x, n-x)=0$. En este caso,

$$
D(x, y)=G(y-x)-\frac{1}{2} G(n-2 x)-\frac{1}{2} G(2 y-n) .
$$

Podemos escribir

$$
D(x, y)=\frac{1}{2}\left(G(y-x)-G(n-2 x) j+\frac{1}{2}(G(y-x)-G(2 y-n))\right.
$$

Aplicando también en este caso dos veces el teorema del valor medio obtene mos

$$
D(x, y)=\frac{1}{2} G^{\prime}(p)(x+y-n)+\frac{1}{2} G^{\prime}(q)(n-x-y)
$$




$$
\begin{aligned}
& =\frac{1}{2}(x+y-n)\left(G^{\prime}(p)-G^{\prime}(q)\right) \\
& =\frac{1}{2}(x+y-n) G^{\prime \prime}(x)(p-q)
\end{aligned}
$$

cierto $\mathrm{p}$ entre $(y-x) \quad y \quad(n-2 x)$, y cierto $q$ entre $(y-x) \quad y$ $(2 y-n), y$ cierto $r$ entre $p y q$. Vemos que $(y-x)$ es el punto medio del segmento definido por $(n-2 x)$ y $(2 y-n)$. Esto implica que los facto res $(x+y-n)$ y $(p-q)$ tienen distinto signo. Entonces, el signo de $D(x, y)$ está dado por el signo de - $G^{\prime \prime}(r)$, el cual es negativo pues en este caso $m=-1$. De este modo ha quedado probado la admisibilidad del par definido por las fórmulas (6) y (7). El hecho que la funcional alcance su valor mínimo para este par sigue directamente de (7).

Ejemplo. Sea $K(x, y)=G(y-x)$ en el cuadrado $[0,1] x[0,1]$, donde $G(t)=-t^{3}$ para $t<0 y G(t)=t^{3}$ para $t \geqslant 0$. Observamos que esta función es diferenciable sólo dos veces en $t=0$. Tenemos,

$$
G^{\prime \prime}(y-x)=\left\{\begin{array}{ccc}
-G(y-x) & \text { si } & y<x \\
G(y-x) & \text { si } & y>x \\
0 & \text { si } & y=x
\end{array}\right.
$$

Vemos que $G^{\prime \prime}(y-x) \geqslant 0$ para todo punto $(x, y)$. Aplicando el teorema preceiente cun $f(x)=1-x$ obtenemos

$$
\begin{aligned}
& f(x)=\frac{1}{2} G(1-2 x)=\left\{\begin{array}{ccc}
-\frac{1}{2}(1-2 x)^{3} & \text { si } & x>\frac{1}{2} \\
\frac{1}{2}(1-2 x)^{3} & \text { si } & x \leqslant \frac{1}{2}
\end{array}\right. \\
& g(y)=\frac{1}{2} G(2 y-1)=\left\{\begin{array}{ccc}
-\frac{1}{2}(2 y-1)^{3} & \text { si } & y<\frac{1}{2} \\
\frac{1}{2}(2 y-1)^{3} & \text { si } & y \geqslant \frac{1}{2}
\end{array}\right.
\end{aligned}
$$


En la siguiente sección mostramos una cierta clase de núcleos pa ra los cuales el problema de Bellman admite soluciones seccionalmente dife renciables. Estas soluciones son obtenilas después de aplicar las fórmulas (6) y (7) en subrectángulos en los cuales $\mathrm{K}_{\mathrm{xy}}$ no cambia de signo a lo largo de sus diagonales y después de exigir continuidad en la solución glo bal.

\section{CIERTA CLASE DE NUCLEOS CON SOLUCIONES SECCIONALMENTE DIFERENCIABLES.}

Por simplicidad suponemos que el problema está dado en el cuadrą do $[0,1] x[0,1]$. Sea $K(x, y)=G(y-x)$, donde $G$ es una función cuya segunda derivada satisface las hipótesis $G^{\prime \prime}(0)=0, G^{\prime \prime}(t)>0$ para todo $t$ en $[-1,0)$ y $G^{\prime \prime}(t)<0$ para todo $t$ en $(0,1]$. Además, sin pérdida de generalidad, podemos suponer que $G(0)=G^{\prime}(0)=0$.

Comenzamos particionando el cuadrado $[0,1] \times[0,1]$ como se indica en la figura siguiente:

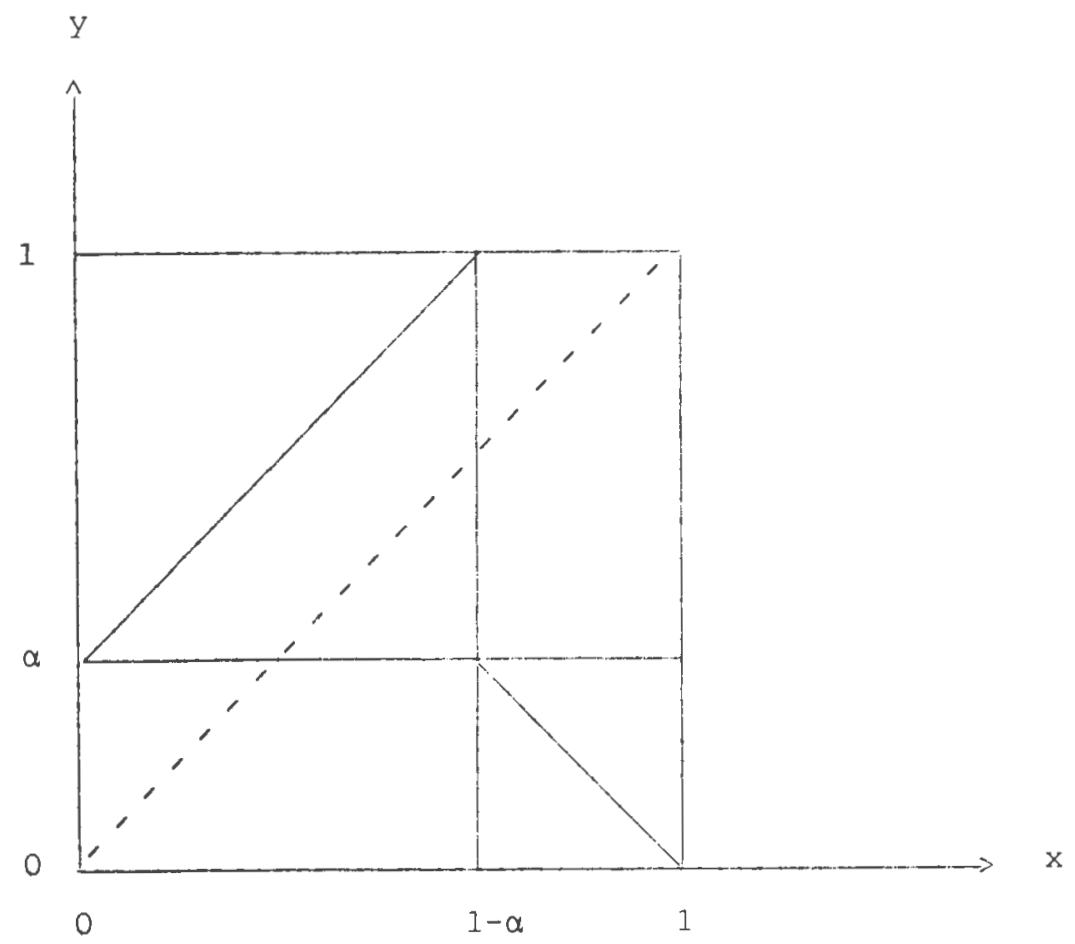


Tenemos que $\mathrm{K}_{\mathrm{xy}}(\mathrm{x}, \mathrm{y})=-\mathrm{G}^{\prime \prime}(\mathrm{y}-\mathrm{x})$. Vemos que si $0<\alpha<\frac{1}{2}$ entonces $G^{\prime \prime}(y-x)$ no cambia de signo en el cuadrado $[1-\alpha, 1] x[0, \alpha]$. Este cuadrado $G^{\prime \prime}(y-x)>0$ si $0<\alpha<\frac{1}{2}$. Bajo esta condición para $\alpha$, aplicando el teorema anterior, el par minimal en este cuadrado está dado por las fórmulas

$$
\begin{aligned}
& f_{2}^{\prime}(x)=K_{x}(x, 1-x)=-G^{\prime}(1-2 x) \\
& g_{1}^{\prime}(y)=K_{y}(1-y, y)=G^{\prime}(2 y-1) \\
& f_{2}(x)+g_{1}(1-x)=K(x, 1-x)=G(1-2 x)
\end{aligned}
$$

De estas fórmulas obtenemos

$$
\begin{aligned}
& f_{2}(x)=\frac{1}{2} G(1-2 x)+C_{3} \\
& g_{1}(y)=\frac{1}{2} G(2 y-1)-C_{3}
\end{aligned}
$$

donde $\mathrm{C}_{3}$ es una constante. En cambio, siempre para $0<\alpha<\frac{1}{2}, \mathrm{G}^{\prime \prime}(\mathrm{y}-\mathrm{x})$ cambia de signo en el cuadrado $[0,1-\alpha] \times[\alpha, 1]$. Sin embargo, la siguiente sucesión de lemas nos permitirá concluir que la utilización de las fórmulas (6) y (7) con $y=\rho(x)=x+\alpha$ conduce a la solución en el cuadrado $[0,1-\alpha] \times[\alpha, 1]$ si además se exige continuidad en los puntos $\mathrm{x}=1-\alpha \mathrm{e} \mathrm{y}=\alpha$. Aplicando estas fórmulas con $\mathrm{y}=f(\mathrm{x})=\mathrm{x}+\alpha$ obtene mos

$$
\begin{aligned}
& f_{1}^{\prime}(x)=K_{x}(x, x+\alpha)=-G^{\prime}(\alpha) \\
& g_{2}^{\prime}(y)=K_{y}(y-\alpha, y)=G^{\prime}(\alpha) \\
& f_{1}(x)+g_{2}(x+\alpha)=K(x, x+\alpha)=G(\alpha) .
\end{aligned}
$$

De estas fórmulas resulta

$$
f_{1}(x)=-G^{\prime}(\alpha) x+C_{1}
$$




$$
g_{2}(y)=G^{\prime}(\alpha) y+C_{2}
$$

donde $\quad C_{1}+C_{2}=G(\alpha)-\alpha G^{\prime}(\alpha)$.

Por su parte la exigencia de continuidad en los puntos $\mathrm{x}=1-*$ e $y=\alpha$, esto es,

$$
\begin{aligned}
& f_{1}(1-\alpha)=f_{2}(1-\alpha) \\
& g_{2}(\alpha)=g_{1}(\alpha)
\end{aligned}
$$

conduce a

$$
f_{1}(1-\alpha)+g_{2}(\alpha)=f_{2}(1-\alpha)+g_{1}(\alpha)
$$

Pero, $\quad f_{2}(1-\alpha)+g_{1}(\alpha)=G(2 \alpha-1)$

Por lo tanto,

$$
f_{1}(1-\alpha)+g_{2}(\alpha)=G(2 \alpha-1)
$$

Reemplazando $f_{1}(1-\alpha)$ y $g_{2}(\alpha)$ en esta última igualdad obtenemos la siguiente ecuación para $\alpha$,

$$
G(2 \alpha-1)-G(\alpha)+G^{\prime}(\alpha)-\alpha G^{\prime}(\alpha)=0
$$

Probaremos más adelante que esta ecuación admite una única raíz entre $0 \mathrm{y} 1 / 2$. Previamente, probamos el siguiente lema.

Lema 1. La función $h(t)=t G^{\prime}(t)-G(t)$ es estrictamente decreciente en el intervalo $(-1,1)$.

Demostración. $\quad h^{\prime}(t)=t G^{\prime \prime}(t)<0$ para todo $t \neq 0$ en el intervalo $(-1,1)$. Además, después de aplicar el teorema del valor medio, se puede 
ver que $h(t)>0$ si $t<0$ y $h(t)<0$ si $t: 0$.

Lema 2. La ecuación (10) tiene una ráíz en el intervalo $\left(0, \frac{1}{2}\right)$.

Demostración. Sea

$$
S(t)=G(2 t-1)-G(t)+G^{\prime}(t)-t G^{\prime}(t) \text {. }
$$

Claramente, $S\left(\frac{1}{2}\right)=\frac{1}{2} G^{\prime}\left(\frac{1}{2}\right)-G\left(\frac{1}{2}\right)=h\left(\frac{1}{2}\right)<0$. Por otra parte, $S(0)=G(-1)>0$. Por lo tanto, existe $\alpha, 0<x<\frac{1}{2}$, tal que $S(x)=0$.

Hemos probado así que la ecuación (10) tiene una luí: en el inter valo $(0,1 / 2)$. Posteriormente, probaremos la unicidad de esta ráiz. El si guiente lema nos garantiza la admisibilidad del par $\left(f_{1}, g_{2}\right)$ en el cuadrado $[0,1-\alpha] \times[\alpha, 1]$.

Lema 3. El par $\left(f_{1}, g_{2}\right)$ es admisible en el cuadrado $[0,1-\alpha] \times[\alpha, 1]$ si a es la raíz de la ecuación (10) en el intervalo $(0,1 / 2)$.

Demostración. Tenemos

$$
K(x, y)-f_{1}(x)-g_{2}(y)=G(y-x)-G^{\prime}(\alpha)(y-x)+\alpha G^{\prime}(\alpha)-G(\alpha) .
$$

Sea $t=y-x$. Entonces, $2 \alpha-1 \leq t \leq 1$ pues $0-x \leq 1-i, \alpha \leq y \leq 1$. Definimos

$$
T(t)=G(t)-G^{\prime}(\alpha) t+\alpha G^{\prime}(\alpha)-G(\alpha) .
$$

Vemos que

$$
\begin{aligned}
& T(y-x)=K(x, y)-f_{1}(x)-g_{2}(y)=G(y-x)-f_{1}(x)-g_{2}(y) \\
& T(\alpha)=0, \\
& T^{\prime}(t)=G^{\prime}(t)-G^{\prime}(\alpha), \quad T^{\prime}(\alpha)=0
\end{aligned}
$$




$$
\begin{aligned}
& I^{\prime \prime}(t)=G^{\prime \prime}(t) \\
& G(2 \alpha-)-f_{1}(1-\alpha)-g_{2}(\alpha)=0=T(2 \alpha-1) \\
& T(0)=\left(x G^{\prime}(\alpha)-G(\alpha)=h(\alpha)-0 .\right.
\end{aligned}
$$

Estamos ahora en condiciones de probar que $T(t)$ - 0 para todo $t$ en $[2 r-1,1]$. Usad do la convexidad de $T$ en el intervalo ... - 1, 0) tere mos para cualquier $t$ en este intervalo que

$$
\begin{aligned}
T(t) & =T(\lambda(2 \alpha-1)+(1-\lambda) 0) \leq \\
& \leq \lambda T(2 \alpha-1)+(1-\lambda) T(0)<0
\end{aligned}
$$

pues $0<\lambda<1, \mathrm{~T}(2 \alpha-1)=0$ y $\mathrm{T}(0)<0$. Considiremos ahora $t$ en el intervalo $(0, a)$. Tenemos,

$$
T(\alpha)-T(t)=T^{\prime}(p)(x-t), \text { cierto } p, \quad t<p<x
$$

Pero, $\quad T(\alpha)=0 \quad y \quad T^{\prime}(p)=G^{\prime}(p)-G^{\prime}(a)>0$ pues $G^{\prime}$ es estrictamente decrecient. ' $n(0,1)$. Por lo tanto, $T(t)$ ' 0 para mio $t$ en el intervalo $(0, a)$. Un razonamiento análogo prueba que tambiér $T$ en una función negativa en $(\alpha, 1)$. Hemos probado de este modo que $\mathrm{I}^{\prime}(t)$ - 0 para todo $t$ en $[2 x-1,1]$. En consecuencia,

$$
k(x, y)=f_{1}(x)+g_{2}(y) \text { en }[0,1-\alpha] x[\alpha, 1]
$$

Coiolario.

El par $\left(f_{1}, g_{2}\right)$ es minimal en el cuadrado $[0,1-i x[a, 1]$ si a satisface la eviación (10), $0<\alpha<\frac{1}{2}$.

Demostración. Se sigue del lema $3 y$ de la ecuación $f_{1}(x)+g_{2}(x+\alpha)=$ $K(x, x+\alpha)$. 
Nuestro jxopósito siguiente es demostrux que el pix (f, g) dádo pur $f=f_{1}$ en $[0,1-\alpha], f=f_{2}$ en $[1-\alpha, 1], g=g_{1}$ en $[0, \alpha]$ $y \quad g=g_{2}$ en $[\alpha, 11$, es minimal en el cuadrado 10,1$] x[0,1]$. Para ello es suficiente probar, en virtud de los teoremas 9 y 10 de [2] o teore $\operatorname{mas} 6$ y 7 de [3] que $f_{1}-f_{2}$ en $[0,1-\alpha], f_{1}=f_{2}$ en $[1-\alpha, 1]$, $g_{1}>g_{2}$ en $[0, \alpha]$ y $g_{1} \leq g_{2}$ en $[\alpha, 1]$. Estirs desigualdades son probadas en los siguientes lemas.

Lema 4. $\mathrm{f}_{1} \geq \mathrm{f}_{2}$ en $[0,1-\alpha] \quad \mathrm{y} \mathrm{f}_{1} \leq \mathrm{f}_{2}$ en $[1-\alpha, 1]$.

Demostración. Sea $d(x)=f_{1}(x)-f_{2}(x)$. Entonc es,

$$
d(x)=-G^{\prime}(\alpha) x+C_{1}-\frac{1}{2} G(1-2 x)-C_{3}
$$

Pero, $\quad \mathrm{f}_{1}(1-\alpha)=\mathrm{f}_{2}(1-\alpha)$. De esta igualda obtenemos

$$
C_{1}-C_{3}=\frac{1}{2} G(2 \alpha-1)+(1-\alpha) G^{\prime}(\alpha)
$$

Luego,

$$
d(x)=-G^{\prime}(\alpha) x-\frac{1}{2} G(1-2 x)+\frac{1}{2} G(21 \quad 1)+y^{\prime}(x)-G^{\prime}(x)
$$

Vemos que

$$
d^{\prime}(x)=-G^{\prime}(\alpha)+G^{\prime}(1-2 x)
$$

Esta identidad será se utilidad en la domostración del lema como veremos nás adelante. Por ahora, investiguemosel signo le d(o). Tenemos

$$
\begin{aligned}
& d(0)=\frac{1}{2} G^{\prime}(\alpha)(1-\alpha)+\frac{1}{2}(G(\alpha)-G(1)) \\
& d(0)=\frac{1}{2} G^{\prime}(\alpha)(1-\alpha)-\frac{1}{2} G^{\prime}(p)(1-\alpha) \\
& d(0)=\frac{1}{2}(1-\alpha)\left(G^{\prime}(\alpha)-G^{\prime}(p)\right)
\end{aligned}
$$


para algún $\mathrm{p}, 0<\alpha<\mathrm{p}<1$. Como $\mathrm{G}^{\prime}$ es decreciente en el intervalo $(0,1)$, podemos concluir de la última igualdad que $d(0)>0$. Ahora busca mos el valor de $d\left(\frac{1-\alpha}{2}\right)$. Tenemos

$$
d\left(\frac{1-\alpha}{2}\right)=\frac{1}{2}\left(G^{\prime}(\alpha)-\alpha G^{\prime}(\alpha)-G(\alpha)+G(2 \alpha-1)\right) .
$$

como a es escogido satisfaciendo la ecuación (10), se sigue que $\mathrm{d}\left(\frac{1-\alpha}{2}\right)=0$. Con la información hasta ahora obtenida estamos en condicio nes de probar que $d(x)>0$ para todo $x$ en $\left(0, \frac{1-\alpha}{2}\right)$. En efecto, pa ra $\mathrm{x}$ en este intervalo tenemos $0<\alpha<1-2 \mathrm{x}$ y entonces

$d^{\prime}(x)=G^{\prime}(1-2 x)-G^{\prime}(\alpha)<0$, lo que implica que para $x$ en este inter valo se cumple $0=d\left(\frac{1-\alpha}{2}\right)<d(x)<d(0)$. Consideremos ahora valores de $x$ en el intervalo $\left(\frac{1-\alpha}{2}, \frac{1}{2}\right)$. Para estos valores se cumple $0<1-2 x<\alpha$ y que $d^{\prime}(x)>0$. Entonces $d(x)>d\left(\frac{1-\alpha}{2}\right)=0$ para $x$ en el intervalo $\left(\frac{1-\alpha}{2}, \frac{1}{2}\right]$. Antes de continuar observamos que d' $\left(\frac{1}{2}\right) \quad 0$. Dado que $d\left(\frac{1-\alpha}{2}\right)=0 y d(1-\alpha)=0$, se sigue que existe un punto $\mathrm{s}$ en el intervalo $\left(\frac{1-\alpha}{2}, 1-\alpha\right)$ tal que $d^{\prime}(s)=0$. Tomando en consideración el signo $d^{\prime}(x)$ en el intervalo $\left(\frac{1-\alpha}{2}, \frac{1}{2}\right]$ vemos que $\frac{1}{2}<s<1-\alpha$. Consideremos finalmente valores de $x \geq \frac{1}{2}$. Para estos valores tenemos $1-2 \mathrm{x} \leq 0$ y entonces $\mathrm{G}^{\prime}(1-2 \mathrm{x})$ es decreciente. Por lo tanto, para $x \geq \frac{1}{2}$ concluimos que $d^{\prime}(x)$ es decreciente. Así hemos obtenido que

$$
d^{\prime}(x)>0 \text { si } \frac{1}{2} \leq x<s, d^{\prime}(s)=0, d^{\prime}(x)<0 \text { si } x \text { s. }
$$

Estos resultados implican que $d(x)>0$ para $\frac{1}{2} \leq x<1-\alpha$ y $d(x) \cdot 0$ para $1-\alpha<x \leq 1$. Con lo cual el lema 4 queda probado.

La gráfjca de la función d luce cumo se muestrá en la figura siguiente: 


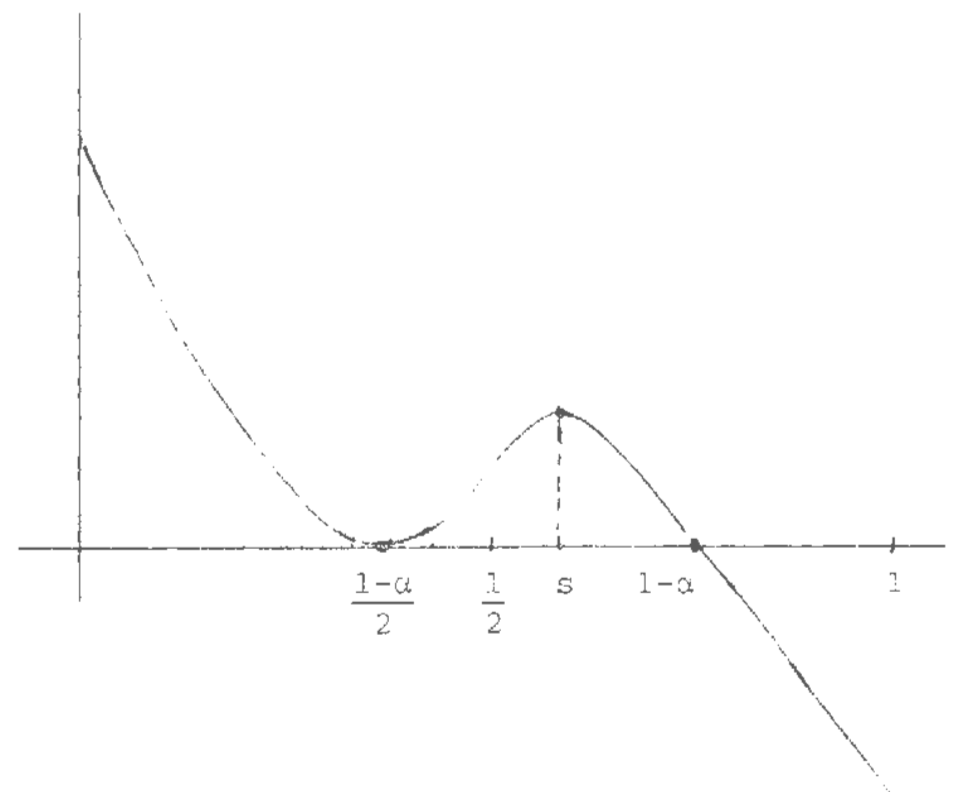

Lema 5. $g_{1} \div 9_{2}$ en $[0, x] \quad y \quad g_{1} \leq g_{2}$ en $[x, 1]$.

Demostración. Sea $e(x)=g_{1}(x)-g_{2}(x)$. Entorces, $e(x)=\frac{1}{2}\left(1(2 x-2)-G^{\prime}(x) x-\frac{1}{2} G(2 .-1)+G^{\prime}(\alpha)\right.$.

lis fäcil ver que $e(1-x)=-d(x)$. For lo tanto, $e(1-x) \therefore 0$ si $0 . x-1-a \quad y \quad e(1-x)$ si $1-\alpha \leq x \leq 1$. se sigue que e $x) \leq 0$

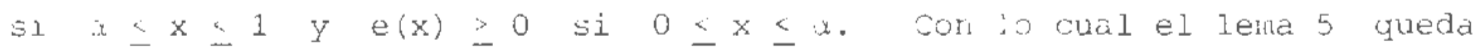
probado.

Pasumos ahora a pebar la uniciad de li räz de la ecuacjor (10) un el intervalo $\left(0, \frac{1}{2}\right)$.

Lema o. Éxiste una únicā râiz de $G(2 t-1)-(t)+G^{\prime}(t)-t G^{\prime}(t)=0$ -. el nutervilo $\left(0, \frac{1}{2}\right)$. 
Demostración.

La existencia de una raíz $\alpha$ en $\left(0, \frac{1}{2}\right)$ para esta ecuación fue probada en el lema 2. Supongamos que exista otra raíz $\beta \neq \alpha$ en este intervalo. Esta raiz nos permite encontrar $\left(\hat{\mathrm{f}}_{1}, \tilde{g}_{2}\right)$ y $\left(\tilde{\mathrm{f}}_{2}, \tilde{g}_{1}\right)$ como pares minimales en $[0,1-\beta] \times[\beta, 1] y[1-\beta, 1] \times[0, \beta]$ respectivamente. Sea $\breve{d}(x)=\tilde{f}_{1}(x)-\tilde{f}_{2}(x)$. Tenemos que

$$
\dot{d}(x)=-G^{\prime}(\beta) x-\frac{1}{2} G^{\prime}(1-2 x)+\frac{1}{2} G^{\prime}(2 \beta-1)+G^{\prime}(\beta)-\beta G^{\prime}(\beta) .
$$

Vemos que $d(x)-\tilde{d}(x)$ es una función lineal. Por lo tanto, existe un único valor $z$ de $x$ tal que $d(z)=\tilde{d}(z)$. Este resultado contradice el hecho que

$$
d\left(\frac{1-\alpha}{2}\right)=d\left(\frac{1-\beta}{2}\right)=0, d(1-\alpha)=d(1-\beta)=0 \text {. }
$$

Queda así probada la unicidad de la raíz $a$.

Teorema.

Sea $K(x, y)=G(y-x)$ en $[0,1] x[0,1]$, donde $G$ es una fun ción cuya segunda derivada satisface $G^{\prime \prime}(0)=0, G^{\prime \prime}(t)>0$ para $t$ en $[-1,0]$ y $G^{\prime \prime}(t)<0$ para $t$ en $(0,1]$. Si además $G^{\prime}(0)=G(0)=0$ entonces el problema de Bellman admite una única solución (f, g), hasta una constante $C$, de funciones seccionalmente diferenciables dadas explíci tamente por las fórmulas:

$$
\begin{aligned}
& f(x)=f_{1}(x)=-G^{\prime}(\alpha) x+C, 0 \leq x \leq 1-\alpha \\
& g(y)=g_{2}(y)=G^{\prime}(\alpha) y+G(\alpha)-\alpha G^{\prime}(\alpha)-C, \alpha \leq y \leq 1 \\
& f(x)=f_{2}(x)=\frac{1}{2} G(1-2 x)-\frac{1}{2} G(2 \alpha-1)-(1-\alpha) G^{\prime}(\alpha)+C, 1-\alpha \leq x \leq 1 \\
& g(y)=g_{1}(y)=\frac{1}{2} G(2 y-1)+\frac{1}{2} G(2 \alpha-1)+(1-\alpha) G^{\prime}(\alpha)-C, 0 \leq y \leq \alpha
\end{aligned}
$$


donde $\alpha$ es la raíz de la ecuación

$$
G(2 \alpha-1)-G(\alpha)+G^{\prime}(\alpha)-i G^{\prime}(\alpha)=0
$$

en el intervalo $\left(0, \frac{1}{2}\right)$. Un valor para $C$ puede ser encontrado usando la con dición de normalización, en cuyo caso queda dado por

$$
C=\frac{1}{2} G(2 \alpha-1)+(1-\alpha) G^{\prime}(\alpha) .
$$

Observación. Si la función $G$ no satisface las hipótesis $G(0)=0$ y $G^{\prime}(0)=0$ entonces el teorema puede aplicarse a la función

$$
H(t)=G(t)-t G^{\prime}(0)-G(0) \text {. }
$$

Obtenida la solución (f, g) para el núcleo H(y - x), la solución para el núcleo $G(y-x)$ queda dada por el par

$$
\left(f(x)-x G^{\prime}(0)+\frac{1}{2} G(0), g(y)+y G^{\prime}(0)+\frac{1}{2} G(0)\right)
$$

Los ejemplos siguientes pretenden mostrar algunos núcleos que per tenecen a la clase de núcleos estudiada en este trabajo.

\section{Ejemplos.}

1) $K(x, y)=(x-y)^{2 n+1}$, donde $n$ es cualquier número natural.

2) $k(x, y)=\operatorname{sen} \frac{I I}{2}(y-x)$

3) $K(x, y)=\int_{-1}^{y-x} e^{-u^{2}} d u$ 


\section{REFERENCIAS.}

[1] R. BELLMAN. Approximation of functions, Bull. Amer. Math. Soc. 76 (1970), 971 .

[2] 0. ROJO. On an Optimization Problem of Bellman, J. Math. Ana1. Appl. 61 (1977), 208-215.

[3] O. ROJO. Una versión continua del problema de transporte, Revista Proyecciones $\mathrm{N}^{\circ}$ 10: 3-23 (Diciembre, 1985).

[4] O. ROJO. Una versión continua del problema de transporte, Resumen de comunicaciones, $2^{\circ}$ Simposio Chileno de Matemática, 22 (Diciembre, 1985). 